\title{
Temperature and electric field tuning of the ferroelectric soft mode in a strained $\mathrm{SrTiO}_{3} / \mathrm{DyScO}_{3}$ heterostructure
}

\author{
C. Kadlec, ${ }^{1}$ V. Skoromets, ${ }^{1}$ F. Kadlec, ${ }^{1}$ H. Němec, ${ }^{1}$ J. Hlinka, ${ }^{1}$ J. Schubert, ${ }^{2}$ G. Panaitov, ${ }^{2}$ and P. Kužel ${ }^{1, *}$ \\ ${ }^{1}$ Institute of Physics, Academy of Sciences of the Czech Republic, Na Slovance 2, 18221 Prague 8, Czech Republic \\ ${ }^{2}$ Institute of Bio and Nanosystems, Research Centre Jülich, JARA-Fundamentals of Future Information Technology, \\ D-52425 Jülich, Germany
}

(Received 12 August 2009; revised manuscript received 27 October 2009; published 25 November 2009)

\begin{abstract}
We studied $\mathrm{THz}$ dielectric spectra of $\mathrm{SrTiO}_{3} / \mathrm{DyScO}_{3}$ epitaxial multilayers with four 50-nm-thick layers of each compound deposited on $\mathrm{DyScO}_{3}$ substrate as a function of temperature and electric field. At room temperature, $\mathrm{SrTiO}_{3}$ is in the paraelectric phase and, due to the in-plane tensile strain within the films, the soft-mode frequency is shifted down to $47 \mathrm{~cm}^{-1}$. The ferroelectric phase transition occurs in the strained $\mathrm{SrTiO}_{3}$ layers around $270 \mathrm{~K}$ and in the ferroelectric phase the soft-mode hardening upon temperature decrease is observed. The soft mode is linearly coupled to an excitation of relaxation type at $10 \mathrm{~cm}^{-1}$ at all temperatures. This relaxation is silent in the paraelectric phase, but it becomes coupled to the polarization below the ferroelectric transition temperature and its strength progressively increases. The recently proposed universal model of soft-mode behavior in strained $\mathrm{SrTiO}_{3}$ films [C. Kadlec et al., J. Phys.: Condens. Matter 21, 115902 (2009)] was generalized to describe the observed spectra at all temperatures and applied fields.
\end{abstract}

DOI: 10.1103/PhysRevB.80.174116

PACS number(s): 77.80.-e, 63.20.-e, 77.55.+f

\section{INTRODUCTION}

Research on dielectric properties of materials in the terahertz spectral range gains importance both for understanding the fundamental processes with spectral signatures in the far infrared and for the development of new applications in the terahertz technology. ${ }^{1}$

Switchable and tunable structures operating in the terahertz range are currently of high interest for the $\mathrm{THz}$ technology with possible applications in future short-range wireless communications. ${ }^{2}$ Various metallic and/or semiconductor metamaterial and photonic structures were proposed to achieve these aims. ${ }^{3-6}$ Electric field tunable dielectrics constitute another class of materials, which may play a significant role in this research. ${ }^{7-9}$

In ferroelectrics, dispersion and absorption related to the ferroelectric soft-mode frequently appear in the upper part of the terahertz range; in addition (or alternatively), a relaxation behavior due to polar disorder is observed in its lower part. These excitations play the key role in the low-frequency dynamics and usually drive the ferroelectric phase transition in these compounds. ${ }^{10}$

Strontium titanate $\left(\mathrm{SrTiO}_{3}, \mathrm{STO}\right)$ is an incipient ferroelectric showing a strong ferroelectric soft mode. In other ferroelectric compounds with perovskite structure, such as $\mathrm{BaTiO}_{3}$, the frequency of the soft phonon mode decreases with temperature in the paraelectric phase to give rise of the ferroelectric phase transition. The ferroelectric transition does not occur in STO single crystals due to quantum fluctuations at low temperatures. ${ }^{11}$ Recently, it has been reported that very thin STO films may exhibit the ferroelectric phase transition provided they are deposited on substrates, showing a suitable lattice-parameter mismatch with STO in order to induce an in-plane tensile strain within the STO film. ${ }^{12}$ The phase transition appears close to room temperature in these films for the lattice mismatch of about $1 \%$, which can be achieved, e.g., on (110) $\mathrm{DyScO}_{3}$ (DSO) substrates. ${ }^{13}$ Such layers show an enhanced tunability of the complex microwave and terahertz dielectric function at room temperature as compared to single crystals and unstrained films. ${ }^{14-16}$

It has been demonstrated that STO/DSO multilayers can be grown on DSO substrates with the aim to increase the total thickness of the strained STO.,16 A strong strain and, as a consequence, high values of the subterahertz dielectric function at room temperature can then be maintained across at least $200 \mathrm{~nm}$ of the total thickness of STO layers. ${ }^{16}$

Recently, a new mode of relaxation type (central mode) has been observed in strained STO films and STO/DSO multilayers. ${ }^{7,17}$ The origin of the coexistence of a soft and central modes close to phase transitions in many perovskite ferroelectrics is still under debate. Recently, an $a b$ initio modeling supported by $\mathrm{THz}$ experimental data has demonstrated its close relation to the anharmonicity of the softmode potential in the ferroelectric $\mathrm{BaTiO}_{3} .{ }^{18,19}$

In this paper, we report on a study of $\mathrm{THz}$ dielectric properties and field-induced tunability of strained STO multilayer in a temperature range of 20-300 K, which encompasses the ferroelectric phase transition. The character of the coupling of the previously reported ${ }^{7,16}$ low-frequency relaxation mode is deduced from low-temperature data.

\section{EXPERIMENTAL DETAILS}

We investigated the temperature-dependent dielectric properties of a multilayer sample (denoted below as 4 $\times 50 \mathrm{~A}$ ) consisting of four STO/DSO bilayers deposited on a DSO single-crystal substrate, which was previously studied at room temperature in Ref. 7; details on the substrate and multilayer preparation are provided therein. The multilayer was prepared by pulsed laser deposition technique and, subsequently, an interdigitated gold electrode structure was deposited on the top STO layer such that it covered about one half of the $1 \mathrm{~cm}^{2}$ sample surface. For comparison, and in order to check the accuracy of the temperature-dependent 
measurements, another (nominally the same) multilayer sample was prepared (sample $4 \times 50 \mathrm{~B}$ ) but it was not covered by the electrode structure.

The $\mathrm{THz}$ time-domain spectroscopy was used to determine the complex permittivity spectra at various temperatures with and without the electric bias. Experiments without bias were carried out on the part of the sample $4 \times 50 \mathrm{~A}$ not covered with electrodes in order to characterize the zero-field permittivity and loss spectra of the STO layers. As pointed out earlier, the accuracy of the determination of the permittivity of thin films is usually rather low. ${ }^{20}$ In addition, in order to limit the probing $\mathrm{THz}$ beam to the bare part of the sample, an aperture of $3 \mathrm{~mm}$ was used in $\mathrm{THz}$ transmission experiments; this may lead to diffraction effects and the lowfrequency part of the $\mathrm{THz}$ spectra (which was subsequently found to be very important for the interpretation) may suffer from larger systematic errors, especially if the sample is handled in the cryostat (problems arise with precise positioning of the sample and reference). For this reason, the sample $4 \times 50 \mathrm{~B}$ was prepared following the same experimental protocol and the temperature-dependent zero-bias $\mathrm{THz}$ transmission experiments were carried out with this sample by using an aperture of $6 \mathrm{~mm}$. In addition, careful preliminary measurements of the substrate thickness, as described in Ref. 17, were performed with both samples in order to reach a high accuracy.

The experiments without bias consisted of two consecutive measurements for each temperature: one of a signal wave form $E_{s}(t)$, using a sample with the thin-film structure, and one of a reference wave form $E_{r}(t)$ with a bare DSO substrate. The ratio of the Fourier transforms of the timedomain wave forms provided the complex $\mathrm{THz}$ transmittance spectrum of the film $t(\omega)=E_{s}(\omega) / E_{r}(\omega)$. The permittivity of the STO film was then retrieved by using the appropriate formula for the thin-film transmittance [see Eq. (3) in Ref. 17].

The electric field-induced changes in the complex dielectric spectra were subsequently determined by measurements of the $\mathrm{THz}$ transmission through the part of the sample 4 $\times 50 \mathrm{~A}$ provided with electrodes and with an applied bias. We used polarized $\mathrm{THz}$ pulses with their electric field parallel to the applied bias field, i.e., perpendicular to the electrode fingers: in this geometry, the electrode structure is transparent to the $\mathrm{THz}$ radiation. Here the reference $\mathrm{THz}$ wave forms $E_{0}(t)$ were measured with the electric field off and the signal wave forms $E_{V}(t)$ were obtained with the applied dc bias; the field-induced changes in the permittivity were then retrieved from the complex transmission function $t^{\prime}(\omega)$ $=E_{V}(\omega) / E_{0}(\omega){ }^{17}$

\section{EXPERIMENTAL RESULTS AND EVALUATION}

Figure 1 shows examples of $\mathrm{THz}$ complex dielectric spectra of unbiased samples. Both the real and imaginary parts of the permittivity decrease with a decreasing temperature. This could be related to a hardening of the soft-mode frequency below the ferroelectric phase transition, which would then occur close to the room temperature. This hypothesis will be proven below by fitting the experimental data with our model.

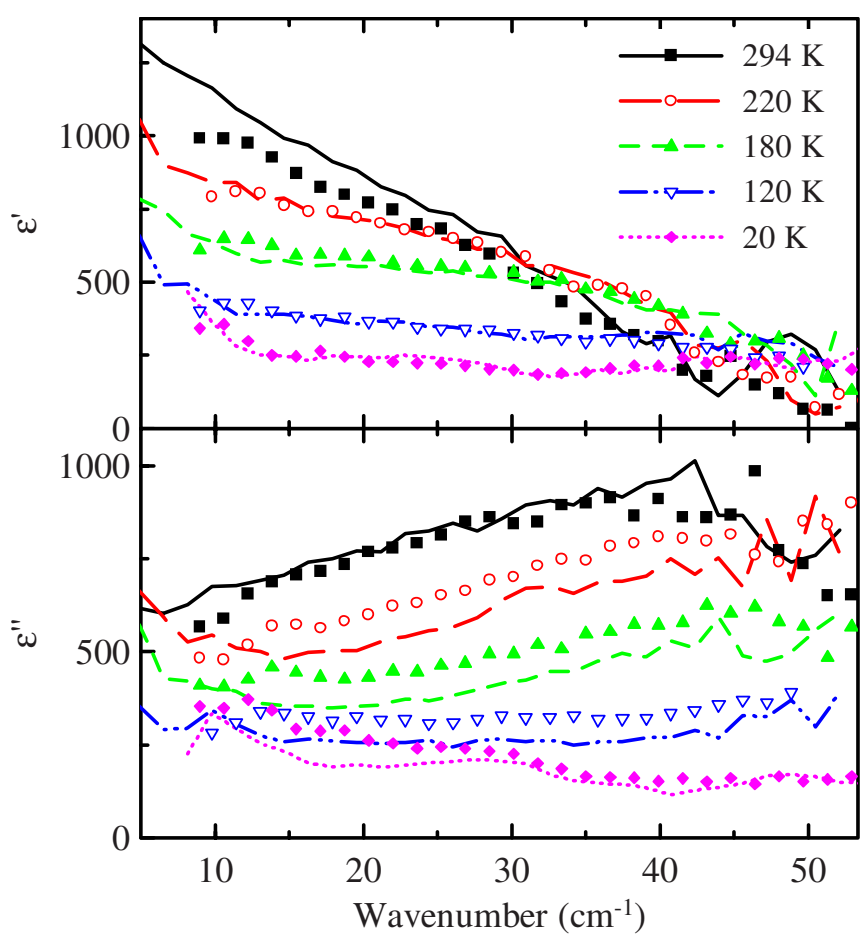

FIG. 1. (Color online) Complex permittivity spectra (without bias) of samples $4 \times 50 \mathrm{~A}$ (symbols) and $4 \times 50 \mathrm{~B}$ (lines) as a function of temperature.

We found a very good agreement between the experimental data obtained with the two investigated samples. This statement and the data plotted in Fig. 1 require a more detailed discussion. As pointed out in Ref. 17, the systematic experimental error in the determination of $\mathrm{THz}$ dielectric spectra in thin films is mostly related to the accuracy of the determination of the substrate thickness due to the so-called phase error. We performed a very careful evaluation of the substrate thickness prior to the thin-film deposition by means of the $\mathrm{THz}$ time-domain spectroscopy of the bare substrates. ${ }^{17,21}$ Nevertheless, small errors on the order of 200 $\mathrm{nm}$ cannot be avoided. In our case, when the bare substrate used for the reference measurement and the substrate with thin-film multilayer have approximately the same thickness, the leading term describing the error in the refractive index $\Delta n$ of the thin film reads as

$$
\Delta n \approx n_{s} \frac{\Delta d_{s}}{d}
$$

where $d$ is the film thickness, $\Delta d_{s}$ is the error in the substrate thickness determination, and $n_{s}$ is the refractive index of the substrate. In this way, an error in the substrate thickness determination of $100 \mathrm{~nm}$ corresponds to $\Delta n \approx 2$; the error in the absorptive index $\Delta \kappa$ is much smaller. Such an offset is then responsible for a systematic error in the measured complex permittivity $\varepsilon=\varepsilon^{\prime}+i \varepsilon^{\prime \prime}$, which can be expressed using the formula

$$
\varepsilon_{\text {new }}=(\sqrt{\varepsilon}-\Delta n)^{2} .
$$

In order to be able to compare easily the data measured on the samples $4 \times 50 \mathrm{~A}$ and $4 \times 50 \mathrm{~B}$, we transformed the per- 


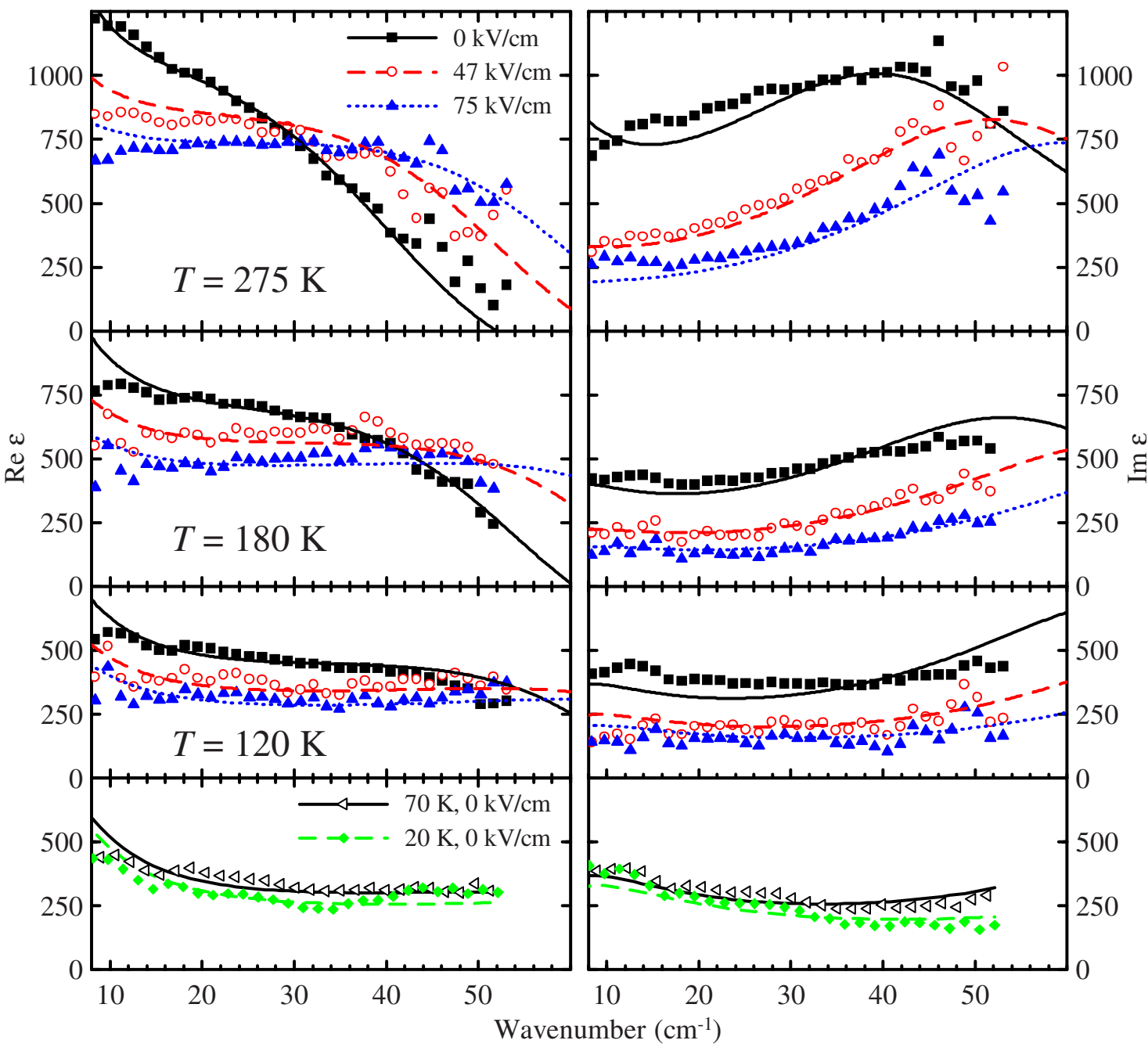

FIG. 2. (Color online) Complex $\mathrm{THz}$ spectra for sample $4 \times 50 \mathrm{~A}$ for several temperatures and bias field. Symbols: experimental data; lines: fit with Eq. (4).

mittivity of the sample $4 \times 50 \mathrm{~A}$ using Eq. (2) with a trial value of $\Delta n=2$. This transformation corresponds to a decrease in $\varepsilon^{\prime}$ by about 70-140 and of $\varepsilon^{\prime \prime}$ roughly by 30-70. Having performed this transformation, we obtained a good agreement between the two samples. This proves an excellent reproducibility in the thin-film elaboration process and demonstrates the state-of-the-art accuracy in our THz measurements. Note, however, that the only reason why we show the transformed data in Fig. 1 is to allow an easy comparison: due to the lack of knowledge of $\Delta d$, it is not possible to remove the systematic error in $\varepsilon$ in this way. All the fits presented in the next section were performed with the asmeasured data.

\section{DISCUSSION}

Figure 2 displays the complex dielectric spectra of sample $4 \times 50 \mathrm{~A}$ as a function of the electric bias for several temperatures. Applying the electric field leads to a hardening of the soft mode as clearly observed in spectra obtained at higher temperatures. In contrast, the tunability of the permittivity is found to be substantially smaller at low temperatures, where the soft-mode frequency is higher even without bias. It has been pointed out previously ${ }^{7,17}$ that a low-frequency excitation (at $\gamma \approx 10 \mathrm{~cm}^{-1}$ ) of relaxation type is observed in the room-temperature spectra. This excitation was shown to be silent, i.e., not directly coupled to the polarization; however, it was linearly coupled to the soft mode (modeled as a damped oscillator with a bare eigenfrequency $\omega_{0}$, damping $\Gamma$, and oscillator strength $f$ ) with a coupling constant $\delta$ $\approx 35 \mathrm{~cm}^{-1}$. The experimental spectra of different STO/DSO multilayers at room temperature were successfully fitted with $^{17}$

$$
\varepsilon(\omega)=\frac{f(1-i \omega / \gamma)}{\left(\omega_{0}^{2}-\omega^{2}-i \omega \Gamma\right)(1-i \omega / \gamma)-\delta^{2}}+\varepsilon_{\infty} .
$$

Within this model, the appearance and strength of the relaxation mode in the $\mathrm{THz}$ dielectric spectra are essentially related to the soft-mode frequency: one finds that the fingerprint of the relaxation practically should not be observed for high soft phonon frequency values $\left(\omega_{0} \geq 90 \mathrm{~cm}^{-1}\right)$, while it should dominate the spectra when the soft-mode frequency is low $\left(\omega_{0} \approx 40 \mathrm{~cm}^{-1}\right)$. We point out that the spectra shown in 


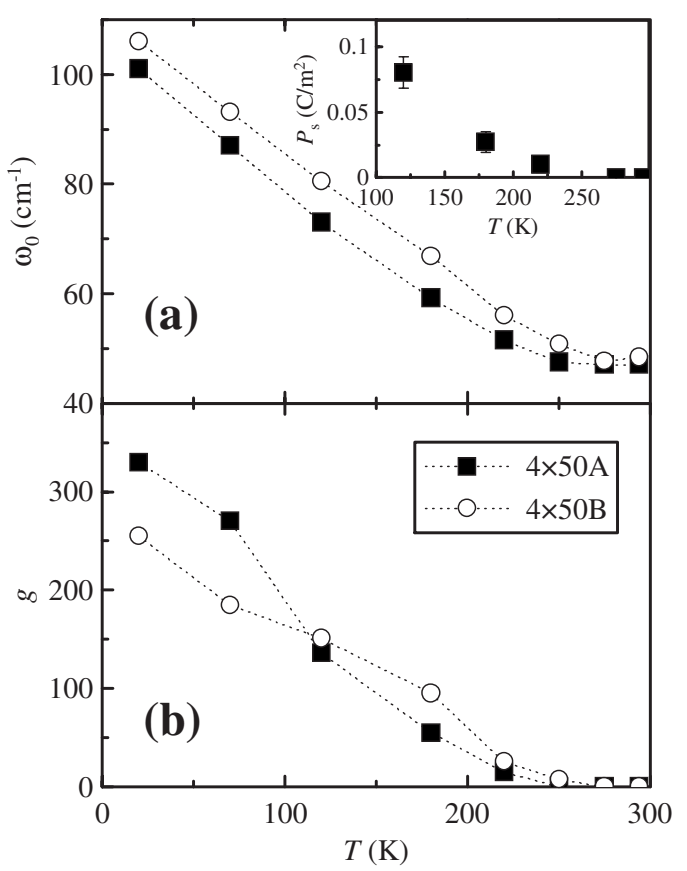

FIG. 3. Temperature dependence of the (a) soft-mode frequency and (b) relaxation strength for both samples studied. Inset: estimation of the temperature dependence of the spontaneous polarization.

Figs. 1 and 2 strongly suggest that this statement is no more valid at low temperatures, where apparently the soft-mode frequency is high while the fingerprints of the relaxation mode are still clearly observed.

Taking into account these facts, we decided to employ a more general formula, ${ }^{22,23}$ which assumes that the bare relaxational mode contributes directly to the permittivity

$$
\varepsilon(\omega)=\frac{f(1-i \omega / \gamma)+g\left(\omega_{0}^{2}-\omega^{2}-i \omega \Gamma\right) \pm 2 \delta \sqrt{f g}}{\left(\omega_{0}^{2}-\omega^{2}-i \omega \Gamma\right)(1-i \omega / \gamma)-\delta^{2}}+\varepsilon_{\infty}
$$

The relaxation mode strength $g$ is proportional to the squared effective charge of the mode. The fits show that it vanishes in the paraelectric phase (as observed in Ref. 17), while it is nonzero in the ferroelectric one and it grows as the temperature decreases below the transition temperature. The sign in the numerator of Eq. (4) describes whether the effective charge of the two modes has the same $(-)$ or opposite $(+)$ sign.

All the spectra measured with and without bias were fitted by the model (4). We performed a global fit, where we assumed that $\Gamma, f, \gamma$, and $\delta$ are known from the roomtemperature data ${ }^{17}$ and that they are temperature and field independent; $g(T)$ was then supposed to be temperature dependent and field independent ${ }^{24}$ and the soft-mode frequency $\omega_{0}(T, E)$ was allowed to be fitted independently for each spectrum. Examples of fits for some spectra are displayed in Fig. 2. More than 50 complex spectra were simultaneously fitted by using the procedure described above; the formula (4) with the sign "+" was found to fit the experimental data

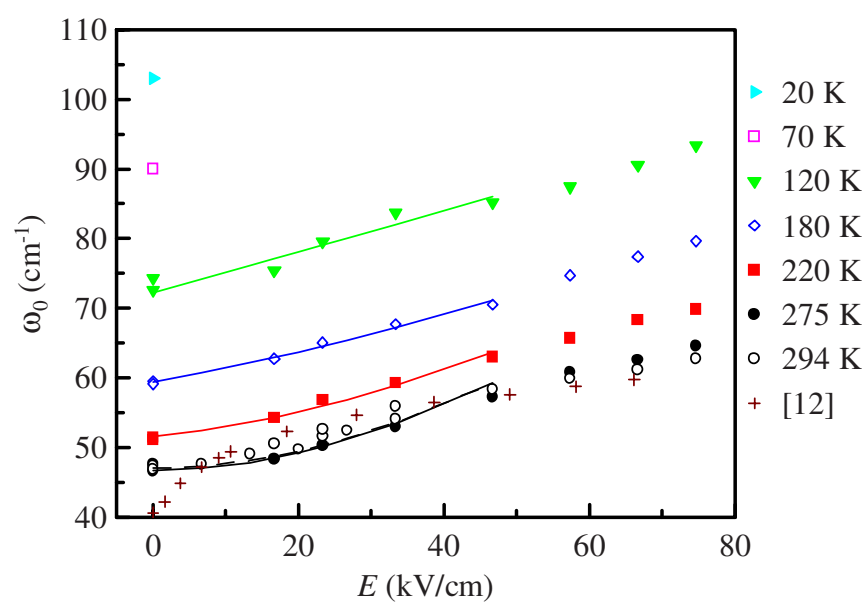

FIG. 4. (Color online) Soft-mode eigenfrequency as a function of the electric bias at several temperatures for sample $4 \times 50 \mathrm{~A}$. Symbols: experiment; lines: fit with Eq. (8), yielding $P_{s}$. Crosses represent an estimation after room-temperature measurements at $10 \mathrm{GHz}$ of a 50-nm-thick STO/DSO in Ref. 12.

quite satisfactorily. This sign corresponds to opposite effective charges of the soft and central modes and implies a destructive interference in the spectral range between the resonance frequencies of the two modes.

The soft-mode frequency without bias and the relaxation strength as a function of temperature are shown in Fig. 3 for both samples. From the data plotted in Fig. 3, the ferroelectric transition temperature appears to occur slightly below the room temperature, say, between 250 and $275 \mathrm{~K}$.

In Fig. 4, we show the electric field tunability of the soft mode at various temperatures as obtained from the fit (measurements with bias were not done at 70 and $20 \mathrm{~K}$ ). It appears that the soft mode significantly hardens when the temperature is decreased but at the same time the tunability in terms of the field-induced change in the soft-mode frequency remains surprisingly high even at low temperatures. To interpret the soft-mode behavior, we consider the simplest form of the Helmholtz free-energy expansion $F$

$$
F=\frac{\omega_{a}^{2}}{2 f \varepsilon_{\mathrm{vac}}} P^{2}+\frac{\beta}{4} P^{4}
$$

where $\varepsilon_{\mathrm{vac}}$ is the vacuum permittivity and the coefficient $\beta$ characterizes the anharmonicity of the soft-mode potential minimum. We denote by $\omega_{0}(T, E)$ the value of the soft-mode eigenfrequency at a given temperature and electric bias. This quantity was not measured in the paraelectric phase above room temperature; nevertheless, following Eq. (5), we formally obtain $\omega_{0}(T, 0)=\omega_{a}(T)$ in this phase. In the ferroelectric phase, $\omega_{a}^{2}$ is renormalized due to the nonvanishing spontaneous polarization $P_{s}$; the value $\omega_{0}(T, 0)$ was experimentally obtained in this phase. In our $\mathrm{THz}$ experiments with an applied bias $E$, we deal with three polarization components, the dc (or low-frequency) ones $P_{s}, \Delta P(E)$ (field-induced), and a weak dynamic one $P_{\mathrm{THz}}$ oscillating at $\mathrm{THz}$ frequencies as the response to the probing pulse. The total in-plane polarization then reads as 


$$
P=P_{s}+\Delta P(E)+P_{\mathrm{THz}} .
$$

The harmonic part of the free energy (5), oscillating at $\mathrm{THz}$ frequencies, then takes the form

$$
F_{\mathrm{THz}}=\frac{\omega_{a}^{2}}{2 f \varepsilon_{\mathrm{vac}}} P_{\mathrm{THz}}^{2}+\frac{3}{2} \beta\left[P_{s}+\Delta P(E)\right]^{2} P_{\mathrm{THz}}^{2} .
$$

Note that thanks to our interdigitated structure, we apply relatively high electric fields which may pole the sample and create regions (in the gaps between electrode fingers) with a nonvanishing macroscopic spontaneous polarization. Owing to the interdigitated character of the electrodes, the adjacent regions are poled with opposite field, i.e., the sign of $P_{s}$ alternates in adjacent electrode gaps (e.g., positive in odd gaps and negative in even gaps between electrode fingers). Note also, however, that the sign of $P_{s}$ and $\Delta P(E)$ is always the same regardless the orientation of the field. Hence, the mixed term $P_{s} \cdot \Delta P(E)$ in Eq. (7) is positive in the whole probed part of the sample and contributes to the electric field-induced soft-mode frequency renormalization

$$
\omega_{0}(T, E) \approx \omega_{0}(T, 0) \sqrt{1+3 \beta\left[\varepsilon_{\mathrm{vac}} f / \omega_{0}^{2}(T, 0)\right]^{3} E^{2}+6 \beta\left[\varepsilon_{\mathrm{vac}} f / \omega_{0}^{2}(T, 0)\right]^{2} P_{s} E} .
$$

The last term under the square root is absent in the paraelectric phase ${ }^{17}$ and in an unpoled sample, while it may significantly contribute to the soft-mode tunability at low temperatures. The best fits of the field dependence of the soft-mode frequency at several temperatures are shown in Fig. 4; the fits were calculated assuming that $\beta$ is temperature independent and $P_{s}$ was a fitting parameter at each temperature. The values of the spontaneous polarization as estimated from the fits are shown in the inset of Fig. 3.

At room temperature, $P_{s}$ vanishes and, in the weak-field limit $(E<35 \mathrm{kV} / \mathrm{cm})$, the soft-mode frequency displays a quadratic growth with the external bias (Fig. 4). The fieldinduced dc polarization grows up to $\Delta P \approx 0.04 \mathrm{C} / \mathrm{m}^{2}$ at $E$ $\approx 35 \mathrm{kV} / \mathrm{cm}$.

Thin STO films grown on YBCO substrates were found to be ferroelectric below $90 \mathrm{~K}$ with a spontaneous polarization of $P_{s} \approx 0.3 \mathrm{C} / \mathrm{m}^{2}$ at helium temperatures. ${ }^{25}$ This value is also comparable to the saturated one found in the ferroelectric phase of $\mathrm{BaTiO}_{3}$ single crystals. ${ }^{26}$ Taking into account these facts, we think that a value of $P_{s} \approx 0.08 \mathrm{C} / \mathrm{m}^{2}$ at 120 K (Fig. 3) stemming from our data is quite reasonable. Nevertheless, this value should be taken with caution as possible contribution of higher-order terms cannot be excluded within the accuracy of our experimental data.

In Ref. 17, a comparison of the soft-mode properties (including the anharmonic coefficient $\beta$ ) has been made for a set of strained samples with different thicknesses of STO layers. These data were obtained by $\mathrm{THz}$ time-domain measurements. It seems then useful to make a comparison of the $\mathrm{THz}$ data, which essentially characterize the soft-mode contribution to the static permittivity with the microwave ones in order to determine whether some additional contribution appears below the phonon range. However, this seems to be difficult with the currently available experimental results, as there is no report characterizing a single sample in both $\mathrm{GHz}$ and $\mathrm{THz}$ ranges. Here we provide a semiquantitative attempt to estimate this difference by comparison of a single 50-nmthick STO layer grown on DSO and characterized in the GHz range $^{12}$ with our multilayer sample (similar comparison can be performed with any of the samples investigated in Ref. 17). A direct comparison is impossible, as the estimated val- ues of the static permittivity from the $\mathrm{GHz}$ data $(\sim 5800)$ (ref. 12) and from the THz data $(\sim 2350)$ (Ref. 17) substantially differ and, consequently, the tunability curves of the dielectric constant are quite different as well. It is not possible to assess whether this difference comes from an additional $\mathrm{GHz}$ contribution or simply from the fact that the samples (and in-plane strains) are different.

An indirect comparison can be made starting from an assumption of the validity of our soft-mode model down to the $\mathrm{GHz}$ frequency range. It is then possible to estimate the variation in the soft-mode eigenfrequency from the roomtemperature tuning curve shown in Fig. 3 in Ref. 12 by using Eq. (4) in the static limit $(\omega \rightarrow 0)$ and with $\delta=35 \mathrm{~cm}^{-1}, f$ $=2.3 \times 10^{6} \mathrm{~cm}^{-2}$, and $g=0$; this is shown in Fig. 4. We observe that for fields higher than $\sim 7 \mathrm{kV} / \mathrm{cm}$, a relatively good agreement with the $\mathrm{THz}$ data is obtained. However, the resulting curve presents a clearly nonquadratic behavior (dip) close to zero bias. This feature can be interpreted as an artifact of our estimation within the soft-mode model and could be attributed to an additional $\mathrm{GHz}$ contribution to the permittivity. A strong contribution to the permittivity in the $\mathrm{GHz}$ range, which was suppressed upon temperature annealing, has been also reported in STO films studied in Ref. 14. Deeper understanding can be obtained only if concerted experiments in $\mathrm{GHz}$ and $\mathrm{THz}$ ranges are carried out.

\section{CONCLUSION}

We determined temperature and electric field dependence of the dielectric permittivity in the $\mathrm{THz}$ range of strained $\mathrm{SrTiO}_{3} / \mathrm{DyScO}_{3}$ multilayers. The ferroelectric phase sets in around $270 \mathrm{~K}$. A general model consisting of a coupled soft mode with a central mode is able to describe all the measured spectra. The effective charge in the central mode has to be taken into account in the ferroelectric phase and is found to be opposite to that of the soft mode. The variation in the soft-mode frequency with temperature and electric field is found to be the key quantity for the overall behavior of $\mathrm{THz}$ dielectric spectra. The electric field tunability of the soft 
mode decreases with decreasing temperature in the ferroelectric phase; however, this decrease is considerably slowed down by a high value of the spontaneous polarization, which renormalizes the effective soft-mode potential. Direct measurements of the spontaneous polarization in the strained samples then would be very helpful in order to confirm the proposed quantitative picture of the soft-mode behavior.

\section{ACKNOWLEDGMENTS}

This work was supported by the Academy of Sciences of the Czech Republic (Project No. AVOZ10100520), by its Grant Agency (Project No. A100100907), and by the Ministry of Education of the Czech Republic (Projects No. LC-512 and No. ME 08109).

\footnotetext{
*Author to whom correspondence should be addressed; kuzelp@fzu.cz

${ }^{1}$ P. Kužel and F. Kadlec, C. R. Phys. 9, 197 (2008).

${ }^{2}$ R. Piesiewicz, T. Kleine-Ostmann, N. Krumbholz, D. Mittleman, M. Koch, J. Schoebel, and T. Kurner, IEEE Antennas Propag. Mag. 49, 24 (2007).

${ }^{3}$ H.-T. Chen, W. J. Padilla, J. M. O. Zide, A. C. Gossard, A. J. Taylor, and R. D. Averitt, Nature (London) 444, 597 (2006).

${ }^{4}$ H.-T. Chen, W. J. Padilla, J. M. O. Zide, S. R. Bank, A. C. Gossard, A. J. Taylor, and R. D. Averitt, Opt. Lett. 32, 1620 (2007).

${ }^{5}$ H.-T. Chen, J. F. O’Hara, A. K. Azad, A. J. Taylor, R. D. Averitt, D. B. Shrekenhamer, and W. J. Padilla, Nat. Photonics 2, 295 (2008).

${ }^{6}$ L. Fekete, F. Kadlec, P. Kužel, and H. Němec, Opt. Lett. 32, 680 (2007).

${ }^{7}$ P. Kužel, C. Kadlec, F. Kadlec, J. Schubert, and G. Panaitov, Appl. Phys. Lett. 93, 052910 (2008).

${ }^{8}$ Q. Zhao, L. Kang, B. Du, H. Zhao, Q. Xie, X. Huang, B. Li, J. Zhou, and L. Li, Phys. Rev. Lett. 101, 027402 (2008).

${ }^{9}$ H. Němec, P. Kužel, F. Kadlec, C. Kadlec, R. Yahiaoui, and P. Mounaix, Phys. Rev. B 79, 241108(R) (2009).

${ }^{10}$ M. E. Lines and A. M. Glass, Principles and Applications of Ferroelectrics and Related Materials (Clarendon, Oxford, 1979).

${ }^{11}$ K. A. Müller and H. Burkard, Phys. Rev. B 19, 3593 (1979).

${ }^{12}$ J. H. Haeni, P. Irvin, W. Chang, R. Uecker, P. Reiche, Y. L. Li, S. Choudhury, W. Tian, M. E. Hawley, B. Craigo, A. K. Tagantsev, X. Q. Pan, S. K. Streiffer, L. Q. Chen, S. W. Kirchoefer, J. Levy, and D. G. Schlom, Nature (London) 430, 758 (2004).
}

${ }^{13}$ P. Irvin, J. Levy, J. H. Haeni, and D. G. Schlom, Appl. Phys. Lett. 88, 042902 (2006).

${ }^{14}$ W. Chang, J. A. Bellotti, S. W. Kirchoefer, and J. M. Pond, J. Electroceram. 17, 487 (2006).

${ }^{15}$ P. Kužel, F. Kadlec, H. Němec, R. Ott, E. Hollmann, and N. Klein, Appl. Phys. Lett. 88, 102901 (2006).

${ }^{16}$ P. Kužel, F. Kadlec, J. Petzelt, J. Schubert, and G. Panaitov, Appl. Phys. Lett. 91, 232911 (2007).

${ }^{17}$ C. Kadlec, F. Kadlec, H. Němec, P. Kužel, J. Schubert, and G. Panaitov, J. Phys.: Condens. Matter 21, 115902 (2009).

${ }^{18}$ I. Ponomareva, L. Bellaiche, T. Ostapchuk, J. Hlinka, and J. Petzelt, Phys. Rev. B 77, 012102 (2008).

${ }^{19}$ J. Hlinka, T. Ostapchuk, D. Nuzhnyy, J. Petzelt, P. Kužel, C. Kadlec, P. Vaněk, I. Ponomareva, and L. Bellaiche, Phys. Rev. Lett. 101, 167402 (2008).

${ }^{20}$ J. Petzelt, P. Kužel, I. Rychetský, A. Pashkin, and T. Ostapchuk, Ferroelectrics 288, 169 (2003).

${ }^{21}$ L. Duvillaret, F. Garet, and J.-L. Coutaz, IEEE J. Sel. Top. Quantum Electron. 2, 739 (1996).

${ }^{22}$ A. S. Barker, Jr., and J. J. Hopfield, Phys. Rev. 135, A1732 (1964).

${ }^{23}$ Y. Girshberg and Y. Yacoby, Solid State Commun. 103, 425 (1997).

${ }^{24}$ Our experimental data are not sufficiently precise to determine unambiguously a possible field-induced variation in $g$ from the fits. Nevertheless, we observed a small general tendency of this parameter to decrease with increasing field at low temperatures.

${ }^{25}$ D. Fuchs, C. W. Schneider, R. Schneider, and H. Rietschel, J. Appl. Phys. 85, 7362 (1999).

${ }^{26}$ W. J. Merz, Phys. Rev. 91, 513 (1953). 